\title{
An ATM Switching Element with programmable capacity
}

\author{
L. Licciardi, M. Peretti, L. Pilati, M. Turolla
}

CSELT, Centro Studi e Laboratori di Telecomunicazione

Via Reiss Romoli, 274 - 10148 Torino, ITALY

Phone: +39112285259 , Fax: +39112287140

e-mail:Maura.Turolla@cselt.it

\begin{abstract}
The paper presents a high performance ATM Switching Element with programmable switching capacity from $8 \times 8$ to $32 \times 32$ links, each at $311 \mathrm{Mbit} / \mathrm{s}$. The $8 \times 8$ component is a single fully CMOS IC, the others can be obtained by properly connecting the $8 \times 8$ basic elements in Multichip Modules. It is shown how to connect them in order to avoid blocking problems, to increase the shared buffer availability and to improve the global system capacity of a factor of 2 or 4 . The expanded Switching Element is a single component in terms of functionality and represents a single-stage in the ATM Switching Fabric. A MCM $16 \times 16$ Switch is also described and its features are presented. Both the components find an industrial application in the Italtel Cross Connect UTXC, which, using these elements, can provide systems from 5 up to $160 \mathrm{Gbit} / \mathrm{s}$.
\end{abstract}




\section{INTRODUCTION}

The Asynchronous Transfer Mode (ATM) has been identified as the backbone technique for transport, multiplexing and switching on the B-ISDN [Pry91]. ATM systems are based on powerful integrated circuits both at the user and at the network management level. Nevertheless in order to preserve investments, flexibility and modularity both at the architectural and at the IC level must be taken into great account. The system manufacturers must provide nodes with different costs, specifications, dimensions and capacity according to the customer needs. Therefore the architectural solutions must be modular and easy to upgrade from medium-low ( $5 \mathrm{Gbit} / \mathrm{s}$ ) to high capacity (several hundred of Gbit/s). As a consequence even the ICs should be as more flexible as possible both in specifications and capabilities. The paper focuses on ATM Switching nodes and mainly on the Switching Element (SE) that is the real core of any ATM Switching Fabric (ASF) [Bal92]. The global switching capability and throughput of the node is highly dependent on the SE speed and capacity. SE most common speed and switching capacity can vary respectively from $155 \mathrm{Mbit} / \mathrm{s}$ to $622 \mathrm{Mbit} / \mathrm{s}$ per any link and from $4 \times 4$ to $32 \times 32$. Anyway depending on specifications and complexity, components are getting more and more complex sometimes reducing yields and increasing costs and power dissipation. Nevertheless working just on increasing switching capability force to reduce other specifications like controllability, debugging, flexibility, programmability that at an industrial viewpoint are sometimes even more important. The solution here presented is focused on a SE of medium complexity $(8 \times 8)$ at $311 \mathrm{Mbit} / \mathrm{s}$ maximum speed per link (BASE8). The technology is CMOS, the complexity is about 700,000 transistors, the power dissipation is $2.3 \mathrm{~W}$ at the working frequency. Using the MCM technique the component can increase its switching capacity from $8 \times 8$ to $32 \times 32$, the $8 \times 8$ SE contains a specific logic, programmable by the microprocessor that allows to program it as a sub-stage of a $16 \times 16$ or $32 \times 32$ element. Architectures and solutions are proposed in order to increase the switching capability as required by the system; any architecture realises a component, seen as a single-stage by the system. An interesting exploitation is the $16 \times 16$ SE (MCM-BASE16), designed by CSELT and realised using the MCM-C technique by IBM. This component, thanks to its $5 \mathrm{Gbit} / \mathrm{s}$ throughput and packaged in a 1073 CCGA, allows to double the switching capability of the complete system.

\section{THE SWITCHING ELEMENT FEATURES}

The $\mathrm{N} \times \mathrm{N}$ switching element can switch $\mathrm{n}$ input links onto $\mathrm{n}$ output links, with $\mathrm{n}=\mathrm{K}^{*} 8$, using basic elements $8 \times 8$, according to the detailed routing information 
laid down in the header of the data stream. All the $n$ input/output links have a 4 bit parallelism, with their own nibble clock, that defines each input/output link frequency and a cell clock. The component internally provides the alignment of the incoming cells with the master internal clock. The cells can be switched to a virtual path or to a distributed path with or without the exclusion of one output link. To avoid traffic congestion and blocking problems many features are developed. A cell loss priority principle is implemented: when the stored cells overcome a threshold, programmable by the microprocessor, low priority cells are discarded, signalling it to the control microprocessor. If back-pressure function has been activated a flag is sent to the previous stage, that will send out from that connection only empty cells. Priority and back-pressure thresholds are programmable. The Switching Element architecture corresponds to a model with output queues, realised with the complete sharing of a large memory properly addressed in order to obtain a spatial switching of the cells towards the outputs. The outgoing cells can be sent out at different speed, programmable link by link.

The component can be configured by an external microprocessor: setting thresholds, output links frequency, forcing empty cell during the initialisation phase. The component is speed programmable up to $311 \mathrm{Mbit} / \mathrm{s}$ per link, both in input links, using the nibble clock, and in output links switching capability of the complete system.

\section{THE $8 \times 8$ SWITCHING ELEMENT}

The Switching Element architecture corresponds to a model with output queues, realised with the complete sharing of a large memory properly addressed in order to obtain a spatial switching of the cells towards the output.

The $8 \times 8 \mathrm{SE}^{1}$ switches 8 input links (ILINK) onto 8 output links (OLINK) as shown in Figure 1 [Tur94]. Starting from the master clock MCK, externally received, the Phase Generator unit creates the internal time base (intClocks). Everything in the device is synchronous with the mentioned time base.

In the DECLINK unit the component internally provides the alignment of the incoming cells with the master internal clock. At the reception of the cell clock signal, the input data are registered in peripheral buffers, that, contain half a cell and let manage even the input link speed, when different from the internal master clock. When one-fourth of a cell is synchronised the reading of the buffer is activated. Beyond the synchronisation as the main function, the DECLINK unit carries out the first checks on the cell: whether the cell has the proper length (64 bytes), whether the routing tag is correct (parity checks), whether the cell is an empty or a test one and whether it has to be discarded for thresholds overcoming (priority or overflow on Shared Buffer).

\footnotetext{
${ }^{1}$ Patent Granted
} 
The Tag Analysis \& Address Generation unit properly address the large memory (Shared Buffer), where the ATM cells are stored. The Rotation Memory adapts the data from the external format (4 bits) to the internal memory format (128 bits) and vice versa.

A Microprocessor Interface handles all information which has to be exchanged between the SE and the external microprocessor such as: diagnostics cares, microprocessor commands, operation and maintenance instructions. Test cells can be extracted from and inserted into the network stream for debugging purposes. The OUTLINK unit adapts the outgoing data from the internal frequency to the programmable output link speed.

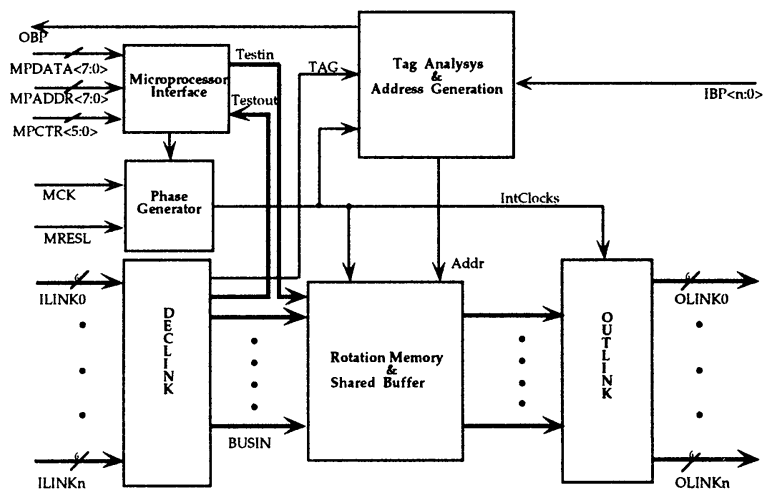

Figure 1. 8 x 8 Switching Element architecture.

High speed and low power dissipation goals strongly drove technology and design choices. In order to achieve such objectives a fully CMOS technology $(0.6 \mu \mathrm{m})$ has been chosen and great care has been taken in the electrical design of critical parts. Thanks to a fully CMOS realisation and to the interesting design solutions the circuit points out with respect to other ATM switching elements, designed in BiCMOS technology, for its power dissipation, for its high working frequency, that implies a very high throughput, for its architectural and designing flexibility and above for the system features addressed to get the component an industrial product.

The $8 \times 8$ circuit works with an internal clock of $77.8 \mathrm{MHz}$ (referring to the UTXC application). We designed full custom the critical structures and we used Standard Cells for glue logic and non critical parts. We customised I/O pads and the internal memories.

The circuit uses special I/O pads ${ }^{2}$ fully CMOS, with a reduced swing $(0 \mathrm{~V}$, $1.5 \mathrm{~V}$ ). In CMOS circuits, dynamic power consumption is proportional to the 
transition frequency, capacitance and square of supply voltage: reducing supply voltage, consequentially, provides significant power saving.

The fast CMOS output driver can work at a rate of $200 \mathrm{Mbit} / \mathrm{s}$, with an eye pattern opened more than $80 \%$. The driver, that uses series termination, is designed with an output impedance equivalent to the line impedance. The output impedance can track the line resistance with an accuracy of $10 \%$. Thanks to the series termination approach (there is dissipation just in transition time), the power consumption is about $30 \mathrm{~mW}$ at $100 \mathrm{MHz}$.

The Shared Memory is a synchronous static RAM (512 words x 128 bits); each word stores one-fourth of an ATM cell, therefore a complete cell is written into four locations of the RAM with contiguous memory addresses. This represents the best trade-off between word size and memory access time constraints for the physical realisation of the system. Particular electrical solutions have been implemented in order to reduce the power dissipation due to the $128 \mathrm{I} / \mathrm{Os}$. Bitlines dynamics was reduced, the SRAM has been clocked only when working and a latching Sense Amplifier ${ }^{3}$ (Figure 2) with a minimum power dissipation has been designed. It works in input with a reduced dynamics of $250 \mathrm{mV}$, with more than $4.5 \mathrm{~V}$ common mode voltage. A specific structure allows to automatically turn-off the sense amplifier biasing and its static power dissipation, it settles in about $2.1 \mathrm{~ns}$ at the worst corner of the process.
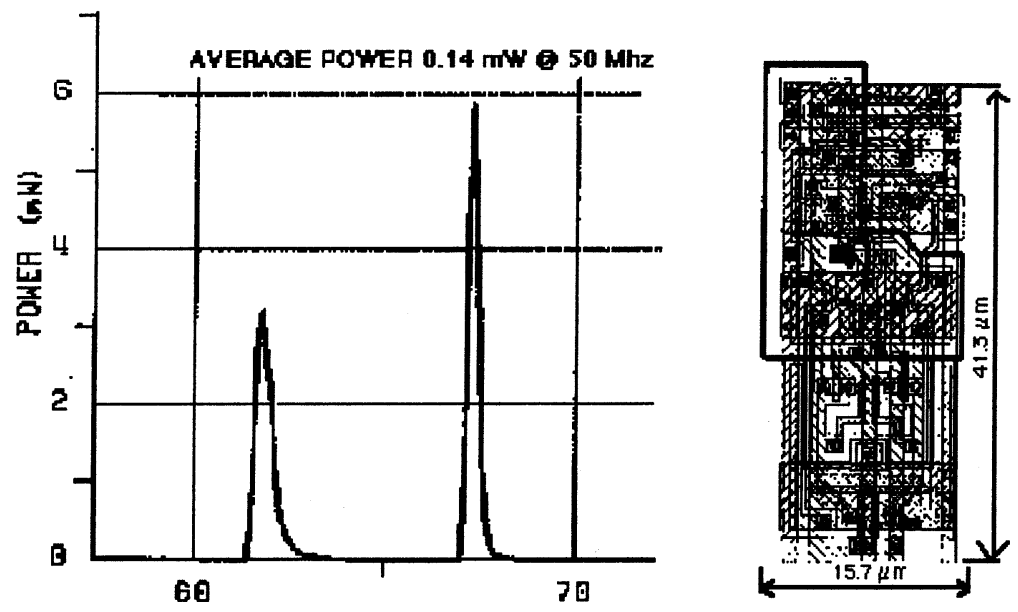

Figure 2. Sense Amplifier Power Dissipation and layout.

Thanks to these solutions the global power dissipation has been limited to $2.3 \mathrm{~W}$ at $77.8 \mathrm{MHz}$ and it has been a key point for a successful usage of the component in the MCM technology. 
The Rotation Memory is a large two-fold shift register structure, implemented as a stack of dynamic shift registers split into 2 planes. To guarantee a high working frequency, the 256 input/output wiring is reduced fitting the layout of this module with the SRAM step.

Particular synchronisation and buffering structures were implemented, by using local latching techniques and balanced length wires, in order to avoid skews among clocks and controls.

\section{THE SWITCHING CAPACITY EXPANSION}

A $8 \times 8 \mathrm{SE}$ can be used to implement a non-blocking $16 \times 16$ or $32 \times 32$ single-stage architecture by using the Multichip module technology.

The routing information is extracted by any stage reading a proper Routing Byte (one for any network stage). In this byte, five bits (called TAG) identify the Output link and two bits specify the Routing Mode (Virtual, Distribution, Distribution with Exclusion). The single-stage architecture is organised in sub-stages of $8 \times 8$ SE and each sub-stage uses a three bits subset (called SUBTAG) extracted from the same TAG.

Using this techniques ${ }^{4}$ a $16 \times 16$ architecture, with four SE organised in two sub-stages (see Figure 3), and a $32 \times 32$ architecture, with twelve SE organised in three sub-stages, can be realised. More complex architectures can be realised just incrementing the number of sub-stages. Using a single TAG for any single-stage architecture is the real key point.

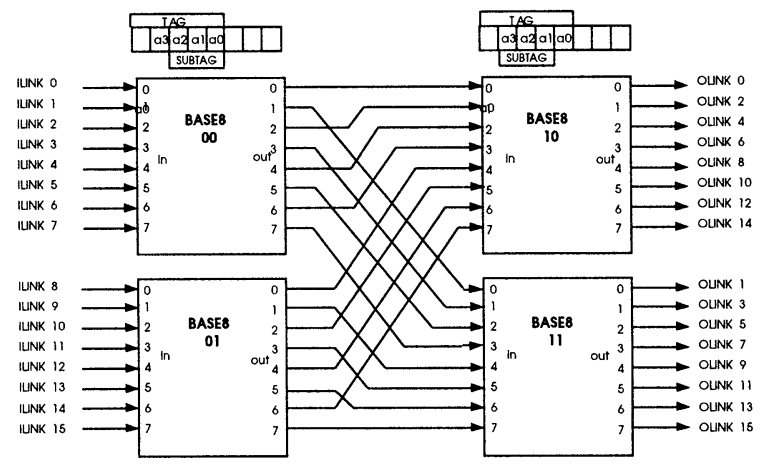

Figure 3. 16 x 16 Single Stage Architecture.

Any base element extracts the proper 3 bits SUBTAG shifting by one.

Two routing modes can be set by the microprocessor: Homogeneous Routing and Mixed Routing Mode. 
In the Homogeneous Routing Mode every sub-stage switches with the same Routing Mode indicated in the Routing Byte. For example in the $16 \times 16$ architecture an ATM cell with Virtual Routing Mode and TAG $=01101$ is routed by the first sub-stage according to SUBTAG $=101$ (out 5 ) and by the second substage according to SUBTAG $=110$ (out 6); the cell is finally routed to link OLINK 13.

In the Mixed Routing Mode only the last sub-stage switches with the Routing Mode indicated in the Routing Byte, whereas all the other sub-stages switch with Distribution Mode towards an odd or even output, using the least significant bit of their SUBTAG. Referring to the previous example, an ATM cell with Virtual Routing Mode and $\mathrm{TAG}=01101$ is routed by the first sub-stage according to SUBTAG = xx1 (odd out) and by the second sub-stage according to SUBTAG = 110 (out 6); the cell is finally routed to link OLINK 13.

\section{THE $16 \times 16$ SWITCHING ELEMENT}

The $16 \times 16 \mathrm{SE}$ is realised with the Multichip Module technique using the architecture and the solutions previously described.

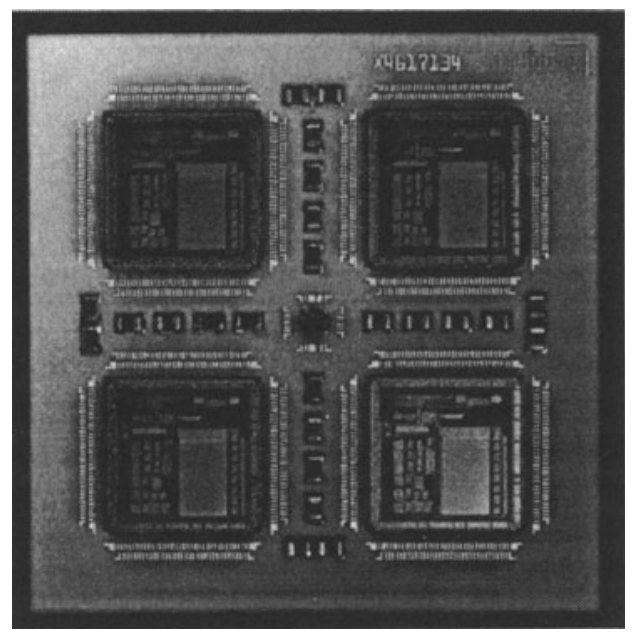

Figure 4. MCM $16 \times 16$ Switching Element substrate top view.

Electrical and thermal simulations were performed in order to analyse and verify signal integrity, simultaneous switching noise (SSN), cross-talk, chip temperatures and power dissipation. The Multichip module is a MCM-C implemented with the Ceramic Column Grid Array (CCGA) technology by IBM, with a substrate made 
of Alumina and Molybdenum (size $43.75 \times 43.75 \mathrm{~mm}^{2}$ ) and a package consisting of 1073 columns for surface mounting technology [Lic96].

The substrate top view is shown in Figure 4. The clock buffer is located in the centre in order to allow balanced length of the clock wires, each BASE8 die has 280 pads distributed on two peripheral rings for wire-bonding, 16 decoupling capacitors and 8 resistors are properly distributed on the substrate with surface mounting technology. Many thermal vias are placed inside the die area in order to provide thermal dissipation towards columns through substrate.

In terms of signal integrity the electrical simulations asserted quite good interconnections for both clock, synchronism and data signals: this is the great advantage of the Multichip module solution compared with the one using four packaged BASE8 components. In fact in the MCM-BASE16 the interconnections between the four $8 \times 8$ components are not subject to the heavy degradation due to the package. The SSN effect was analysed in the worst case when all signals are switching except one, the maximum peak-to-peak swing due to SSN is less than $5 \%$ of the complete swing $(1.5 \mathrm{~V})$, Figure 5.

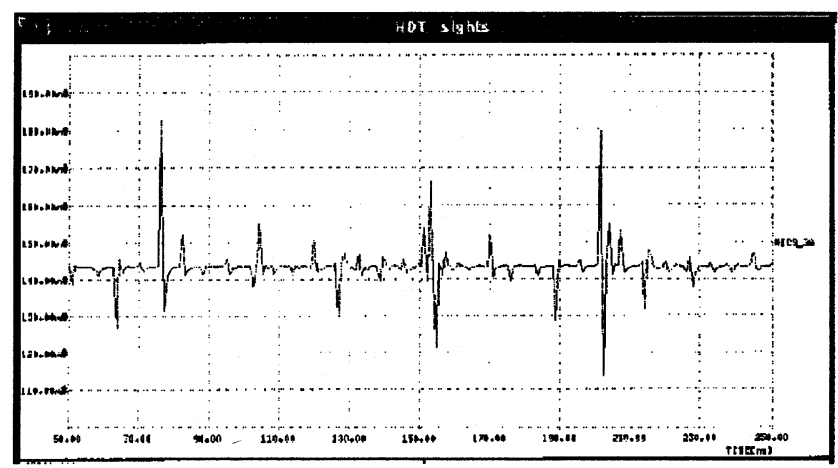

Figure 5. SSN on a BASE8 output link.

No problems on cross-talk analysis were detected. The external interconnections (from die pads towards $\mathrm{V} / \mathrm{O}$ pins) were designed as short as possible to minimise impedance mismatch.

The substrate cross-section is organised in 11 layers: 5 signal layers for routing and redistribution (lines with $50 \Omega$ impedance), 4 power supply layers (implemented as a meshed plane), the top and bottom layers for die attach and columns location.

The package is an array of $33 \times 33$ pins (335 for signals, 442 for GND and 296 for $V_{D D}$ and $V_{L}$ power supplies). These pins are columns of $\mathrm{Pb} / \mathrm{Sn}$ with $1.27 \mathrm{~mm}$ pitch and $2.2 \mathrm{~mm}$ height for surface mounting technology.

Due to the high density of power dissipation (2.3 W for BASE8 and $0.4 \mathrm{~W}$ for clock buffer) and the high thermal constraints (free convection at $0.25 \mathrm{~m} / \mathrm{s}$ and ambient air temperature of $40^{\circ} \mathrm{C}$ ) a Thermalloy aluminium heat-sink is attached 
over the non-hermetic lid of substrate in order to provide thermal dissipation; moreover the high number of power columns used as thermal columns improves dissipation from the MCM towards the board.

The 3D thermal simulation shows that a forced air flow at $1 \mathrm{~m} / \mathrm{s}$ is strictly recommended in order to guarantee a junction temperature less than $100{ }^{\circ} \mathrm{C}$. In terms of boards both the $8 \times 8$ and the $16 \times 16$ (Figure 6) are single board solutions. Complete compatibility is guaranteed in terms of connections, controls and back-plane insertion.

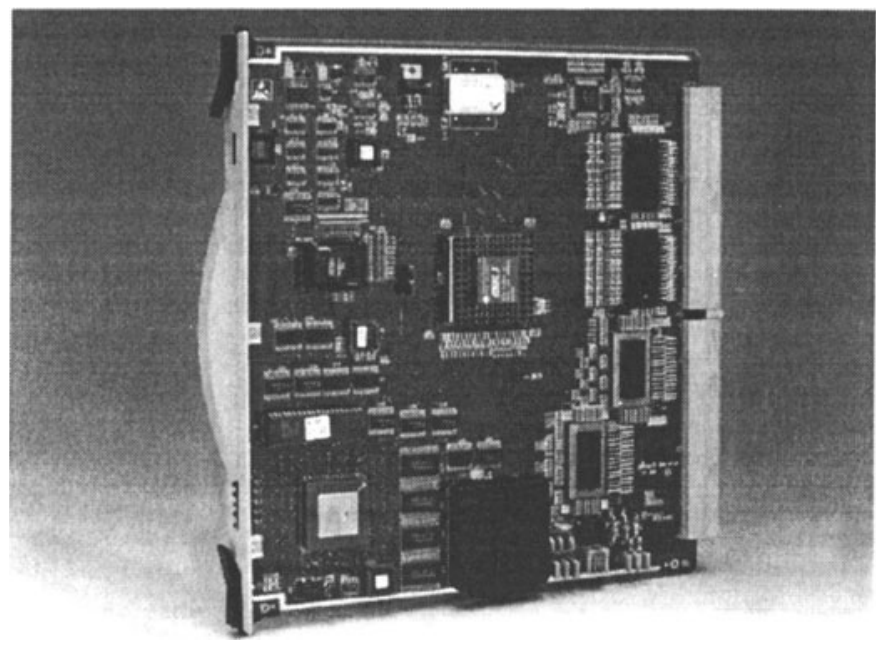

Figure 6. 16 x 16 Switching Board.

\section{UTXC SYSTEM APPLICATION}

The UTXC reference functional architecture reflects a partitioning that allows a simple and flexible node sizing. In fact it can grow from $5 \mathrm{Gbit} / \mathrm{s}$ to $160 \mathrm{Gbit} / \mathrm{s}$ by adding modules [Co194, Bal95].

The overall functionality of the ATM switching system, described above, is realised by connecting a limited number of basic modular chips, with the only addition of external SRAMs, one or more microcontrollers and commercial line driver ECL buffers.

The circuits, designed by CSELT and Italtel, are inserted in the ATM Switching architecture of the Italtel UTXC node (Figure 7). The peripheral part (Exchange Termination), representing the ATM interface of the Broadband Termination Unit (BTU) acts on both the incoming and outgoing cells, the CHP and DPRC components being bi-directional. 
The input ATM cell flow is subjected to UNI/NNI ATM Layer operations, including Usage/Network Parameter Control and VPI/VCI switching, fully complying to ITU-TS Recs. Then, the ATM cell format is transformed into the proprietary one, adding the internal information used inside the system for routing and handling the cell, while adapting the external bit rate (i.e., $155.52 \mathrm{Mbit} / \mathrm{s}$ ) to the internal one (i.e., $311.04 \mathrm{Mbit} / \mathrm{s}$, due to the different cell format and internal speed advantage). The proprietary cell is then duplicated, after having been marked with a time stamp value, and each copy is sent independently to the corresponding part of the ASF, that is one of the two identical parts forming the duplicated ASF. Within the ASF, the cell is routed according to a random distribution algorithm to the ASF output port identified by the routing fields contained in the cell proprietary header.

Figure 7 shows the complete $160 \mathrm{Gbit} / \mathrm{s}$ configuration, using 2 stages of $8 \times 8 \mathrm{SE}$ in the ATM Peripheral Modules (APM) and 3 stages of $16 \times 16 \mathrm{SE}$ in the central stages; the first stages are routed in distribution mode the others in virtual mode in order to send the cells to the required connection.

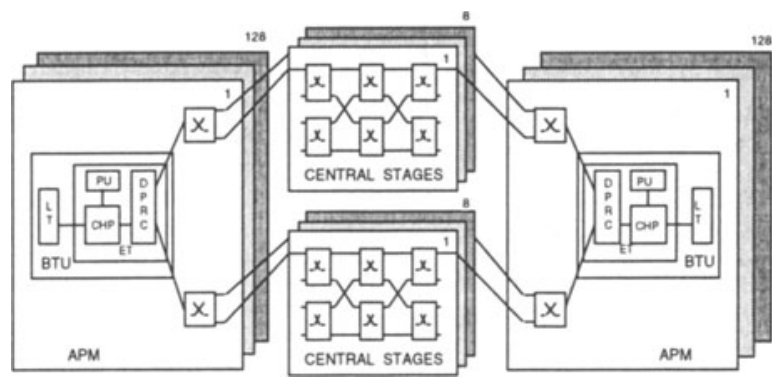

Figure 7. UTXC Switching Network.

The $10 \mathrm{Gbit} / \mathrm{s}$ and $20 \mathrm{Gbit} / \mathrm{s}$ are obtained with 3 stages using respectively the $8 \times 8$ or the $16 \times 16 \mathrm{SE}$ in the central stage. After the ASF output port the duplicated cell is properly selected and the cell sequence is rebuilt thanks to a maximum delay equalisation mechanism. Finally, the internal cell format is transformed into the ATM external one by removing the proprietary fields, while adapting the speed to the external one.

\section{CONCLUSIONS}

ATM tight requirements both in terms of speed and flexibility force a very good mastery of technology at any level (IC, packaging techniques, boards, mechanics, interconnections and so on). An Integrated Design methodology is required to provide good industrial systems. The described ATM Switching Element is a clear example how system requirements, IC design, new packaging solutions and 
architectural issues can be merged in order to provide efficient and modular solutions for the high demanding Telecom business.

The availability of components with different capacity and cost $(8 \times 8$, $16 \times 16 \ldots$ ) well fits the different application requirements both in terms of system costs, integration and global throughput.

\section{ACKNOWLEDGEMENTS}

We wish to sincerely thank all the Italtel and CSELT researchers involved in the UTXC project and IBM Montpellier Technologies for the successful co-operation in the MCM-BASE 16 development.

\section{REFERENCES}

[Bal92] G. Balboni, A. Bellman, M. Collivignarelli, A. Daniele, M. Gandini, L. Licciardi, L. Verri, (October 1992) Key issues in designing a flexible ATM switch, ISS 1992, Yokohama.

[Bal95] G. Balboni, M. Collivignarelli, L. Licciardi, A. Paglialunga, G. Rigoglio, F. Zizza, (April 1995) From transport backbone to service platform: facing the broadband switch evolution, ISS 1995, Berlin.

[Co194] M. Collivignarelli, A. Daniele, P. De Nicola, L. Licciardi, M. Turolla, A. Zappalorto, (November 1994), A complete set of VLSI circuits for ATM Switching, Globecom 1994, San Francisco.

[Lic96] L.Licciardi, M. Peretti, L. Pilati, J. Ichai, and F. Martin, (1996), A set of Multichip Modules for High Speed Telecom Applications, Microcircuits \& Electronic Packaging Journal, Vol. 19, Number 4, Fourth Quarter, 1996.

[Pry91] M. de Prycker, (1991) Asynchronous Transfer Mode, Ellis Hornwood Ltd.

[Tur94] M. Turolla, S. Claretto, M. Fassino, L. Licciardi, (August 1994), A low power ATM Switching Element for Broadband Applications, ITS 1994, Rio de Janeiro.

\section{BIOGRAPHY}

Luigi Licciardi was born in Vercelli, Italy, in 1956. He received his degree in Electronic Engineering from the "Politecnico di Torino" in 1980. Since 1983 he has been with CSELT where he started to work on VLSI digital circuit design for Telecom applications. His main activity is now the development of circuits and systems for ATM and Fast Packet Switching. Since 1991 he is in the Switching and Network Service Department and he is now in charge of the Switching and Hardware Technology Line. 
Dr. Licciardi is the author of several papers on VLSI circuit design, CAD development, switching systems and new technologies for Telecommunications and is named as inventor in several basic patents dealing with the mentioned topics.

Mauro Peretti was born in Turin Italy, in 1960 . He received his degree in Electronic Engineering from the "Politecnico di Torino". He joined CSELT in 1990, working in the Switching System Department on advanced switching techniques and high speed interconnections. He was involved in the RACE BLNT (Broadband Local Network Technology) project of the ATM switching network. At the present, he is a Member of Technical Staff in the Switching Techniques Research Unit, where multichip modules are developed for ATM applications.

Luciano Pilati was born in Turin, Italy, in 1950. He joined CSELT in 1969, graduated in Dipl. Eng. (Industrial Telecommunications) in the Switching Systems Department. Since then, he has been working in the advanced switching techniques field, where he has been involved in design of switching networks (UT100 system), a high speed space and time switching project, and laboratory trials. He has recently working on high speed and optical interconnection in switching systems. At present, he is a Member of Technical Staff in the Switching Techniques Research Unit.

Maura Turolla graduated in Electronic Engineering from the "Politecnico di Torino" in 1982. Since 1983 she has been with CSELT where she started working on VLSI digital circuit design and testing, mainly for signal processing and switching applications. Her main activity currently is the development of circuits for Telecom and ATM applications, focusing her research on low power design and synthesis. Since 1991 she is in the Network Service Department, in the Switching and Hardware Technology Line.

Dr. Turolla has published several papers in the area of VLSI circuit design and is inventor on several patents related to the mentioned topics. 